\title{
Oscillation Criteria for Functional Dynamic Equations with Nonlinearities Given by Riemann-Stieltjes Integral
}

\author{
Yuangong Sun ${ }^{1}$ and Taher S. Hassan ${ }^{2,3}$ \\ ${ }^{1}$ School of Mathematical Sciences, University of Jinan, Jinan, Shandong 250022, China \\ ${ }^{2}$ Department of Mathematics, Faculty of Science, University of Hail, Hail 2440, Saudi Arabia \\ ${ }^{3}$ Department of Mathematics, Faculty of Science, Mansoura University, Mansoura 35516, Egypt
}

Correspondence should be addressed to Yuangong Sun; sunyuangong@163.com

Received 9 January 2014; Revised 24 March 2014; Accepted 28 March 2014; Published 23 April 2014

Academic Editor: Tongxing Li

Copyright (C) 2014 Y. Sun and T. S. Hassan. This is an open access article distributed under the Creative Commons Attribution License, which permits unrestricted use, distribution, and reproduction in any medium, provided the original work is properly cited.

We present new oscillation criteria for the second order nonlinear dynamic equation $\left[r(t) \phi_{\gamma}\left(x^{\Delta}(t)\right)\right]^{\Delta}+q_{0}(t) \phi_{\gamma}\left(x\left(g_{0}(t)\right)\right)+$ $\int_{a}^{b} q(t, s) \phi_{\alpha(s)}(x(g(t, s))) \Delta \zeta(s)=0$ under mild assumptions. Our results generalize and improve some known results for oscillation of second order nonlinear dynamic equations. Several examples are worked out to illustrate the main results.

\section{Introduction}

In this paper, we are concerned with the oscillatory behavior of the second order nonlinear functional dynamic equation with $\gamma$-Laplacian and nonlinearities given by RiemannStieltjes integral

$$
\begin{aligned}
& {\left[r(t) \phi_{\gamma}\left(x^{\Delta}(t)\right)\right]^{\Delta}+q_{0}(t) \phi_{\gamma}\left(x\left(g_{0}(t)\right)\right)} \\
& \quad+\int_{a}^{b} q(t, s) \phi_{\alpha(s)}(x(g(t, s))) \Delta \zeta(s)=0,
\end{aligned}
$$

where the time scale $\mathbb{T}$ is unbounded above; $\phi_{\gamma}(u):=|u|^{\gamma-1} u$, $\gamma>0 ; \alpha \in C[a, b)_{\widehat{\mathbb{T}}}$ with $-\infty<a<b \leq \infty$ is strictly increasing; $\hat{\mathbb{T}}$ is a time scale; $r$ is a positive $\mathrm{rd}-$ continuous function on $\mathbb{T} ; q_{0}$ and $q$ are nonnegative rdcontinuous functions on $\mathbb{T}$ and $\mathbb{T} \times \hat{\mathbb{T}}$ with $q_{0}, q \neq \equiv$; the functions $g_{0}: \mathbb{T} \rightarrow \mathbb{T}$ and $g: \mathbb{T} \times \widehat{\mathbb{T}} \rightarrow \mathbb{T}$ are rd-continuous functions such that $\lim _{t \rightarrow \infty} g_{0}(t)=\infty$ and $\lim _{t \rightarrow \infty} g(t, s)=$ $\infty$ for $t \in \mathbb{T}$ and $s \in \widehat{\mathbb{T}}$.

Both of the following two cases:

$$
\int_{t_{0}}^{\infty} r^{-1 / \gamma}(t) \Delta t=\infty, \quad \int_{t_{0}}^{\infty} r^{-1 / \gamma}(t) \Delta t<\infty
$$

are considered. We define the time scale interval $\left[t_{0}, \infty\right)_{\mathbb{T}}$ by $\left[t_{0}, \infty\right)_{\mathbb{T}}:=\left[t_{0}, \infty\right) \cap \mathbb{T}$. By a solution of (1) we mean a nontrivial real-valued function $x \in C_{\mathrm{rd}}^{1}\left[T_{x}, \infty\right)_{\mathbb{T}}, T_{x} \geq$ $t_{0}$, which has the property that $r \phi_{\gamma}\left(x^{\Delta}\right) \in C_{\mathrm{rd}}^{1}\left[T_{x}, \infty\right)$ and $x$ satisfies (1) on $\left[T_{x}, \infty\right)_{\mathbb{T}}$, where $C_{\text {rd }}$ is the space of rdcontinuous functions. The solutions vanishing identically in some neighborhood of infinity will be excluded from our consideration. A solution $x$ of (1) is said to be oscillatory if it is neither eventually positive nor eventually negative; otherwise it is nonoscillatory.

Not only does the theory of the so-called "dynamic equations" unify theories of differential equations and difference equations, but also it extends these classical cases to cases "in between," for example, to the so-called q-difference equations when $\mathbb{T}=q^{\mathbb{N}_{0}}$ (which has important applications in quantum theory (see [1])) and can be applied in different types of time scales like $\mathbb{T}=h \mathbb{Z}, \mathbb{T}=\mathbb{N}_{0}^{2}$, and $\mathbb{T}=\left\{H_{n}\right\}$ the set of harmonic numbers. In this work knowledge and understanding of time scales and time scale notation is assumed; for an excellent introduction to the calculus on time scales, see Bohner and Peterson [2-4].

In the last few years, there has been increasing interest in obtaining sufficient conditions for the oscillation/nonoscillation of solutions of different classes of 
dynamic equations; we refer the reader to [5-25] and the references cited therein. Recently, Erbe et al. [26] considered

$$
\left(r(t)\left(x^{\Delta}(t)\right)^{\gamma}\right)^{\Delta}+\sum_{i=0}^{n} q_{i}(t) \Phi_{\alpha_{i}}\left(x\left(g_{i}(t)\right)\right)=0
$$

on an arbitrary time scale $\mathbb{T}$, where $\gamma$ is a quotient of odd positive integers and $\Phi_{\alpha_{i}}(u)=|u|^{\alpha_{i}}$ sgn $u$ with $\alpha_{i}>0$ and $\alpha_{0}=\gamma, r$ is a positive rd-continuous function on $\mathbb{T}, q_{i}, i=$ $0,1,2, \ldots, n$, are nonnegative rd-continuous functions on $\mathbb{T}$, and $g_{i}: \mathbb{T} \rightarrow \mathbb{T}, i=0,1,2, \ldots, n$, satisfy $\lim _{t \rightarrow \infty} g_{i}(t)=\infty$. In [26], some oscillation criteria have been established when $g_{i}(t) \equiv \tau(t), i=1,2, \ldots, n, \tau(t) \leq t$, and $\tau$ is nondecreasing and delta differentiable with $\tau o \sigma=\sigma o \tau$ on $\left[t_{0}, \infty\right)_{\mathbb{T}}$. In this paper, we will establish oscillation criteria for the more general equation (1) under mild assumptions on the time scale $\mathbb{T}$ and the time delay. Note that (1) not only contains a $p$-Laplacian term $\gamma>0$ and the advanced/delayed function $g$, but also allows an infinite number of nonlinear terms and even continuous nonlinearities determined by the function $\zeta$.

\section{Main Results}

Throughout this paper, we denote

$$
\begin{array}{cl}
d_{+}(t):=\max \{0, d(t)\}, & d_{-}(t):=\max \{0,-d(t)\}, \\
\lambda(u):=\int_{u}^{\infty} r^{-1 / \gamma}(u) \Delta u, & R(v, u):=\int_{u}^{v} r^{-1 / \gamma}(s) \Delta s .
\end{array}
$$

Lemma 1. Assume that

$$
\int_{t_{0}}^{\infty} r^{-1 / \gamma}(t) \Delta t=\infty
$$

or

$$
\begin{gathered}
\int_{t_{0}}^{\infty} r^{-1 / \gamma}(t) \Delta t<\infty \\
\int_{t_{0}}^{\infty} r^{-1 / \gamma}(v)\left[\int_{t_{0}}^{v} Q_{1}(u) \Delta u\right]^{1 / \gamma} \Delta v=\infty,
\end{gathered}
$$

where

$$
\begin{aligned}
Q_{1}(w):= & q_{0}(w) \lambda^{\gamma}\left(g_{0}(w)\right) \\
& +\int_{a}^{b} q(w, s)\left[\lambda^{\alpha(s)}(g(w, s))\right] \Delta \zeta(s) .
\end{aligned}
$$

If (1) has a positive solution $x$ on $\left[t_{0}, \infty\right)_{\mathbb{T}}$, then there exists a $T \in\left[t_{0}, \infty\right)_{\mathbb{T}}$, sufficiently large, so that

$$
x^{\Delta}(t)>0, \quad\left[r(t) \phi_{\gamma}\left(x^{\Delta}(t)\right)\right]^{\Delta} \leq 0, \quad t \in[T, \infty)_{\mathbb{V}} .
$$

Proof. Pick $T \in\left[t_{0}, \infty\right)_{\mathbb{\mathbb { T }}}$ sufficiently large such that $(t)>$ $0, x\left(g_{0}(t)\right)>0$, and $x(g(t, s))>0$ on $[T, \infty)_{\mathbb{T}} \times[a, b]_{\widehat{\mathbb{T}}}$. From (1), we have, for $t \in[T, \infty)_{\mathbb{T}}$,

$$
\begin{aligned}
{\left[r(t) \phi_{\gamma}\left(x^{\Delta}(t)\right)\right]^{\Delta}=} & -q_{0}(t)\left[x\left(g_{0}(t)\right)\right]^{\gamma} \\
& -\int_{a}^{b} q(t, s)[x(g(t, s))]^{\alpha(s)} \Delta \zeta(s) \leq 0 .
\end{aligned}
$$

Then $r \phi_{\gamma}\left(x^{\Delta}\right)$ is nonincreasing on $[T, \infty)_{\mathbb{T}}$, and $x^{\Delta}$ is of definite sign eventually. We claim that $x^{\Delta}$ is eventually positive. If not, $x^{\Delta}$ is eventually negative; that is, there exists $T_{1} \geq T$ such that $x^{\Delta}(t)<0$ for $t \geq T_{1}$.

First, we assume (5) holds. Using the fact that $r \phi_{\gamma}\left(x^{\Delta}\right)$ is nonincreasing, we obtain, for $t \in\left[T_{1}, \infty\right)_{\mathbb{T}}$,

$$
\begin{aligned}
x(t) & =x\left(T_{1}\right)+\int_{T_{1}}^{t} \phi_{\gamma}^{-1}\left[r(u) \phi_{\gamma}\left(x^{\Delta}(u)\right)\right] r^{-1 / \gamma}(u) \Delta u \\
& <x\left(T_{1}\right)+\phi_{\gamma}^{-1}\left[r\left(T_{1}\right) \phi_{\gamma}\left(x^{\Delta}\left(T_{1}\right)\right)\right] \int_{T_{1}}^{t} r^{-1 / \gamma}(u) \Delta u .
\end{aligned}
$$

Hence, by (5), we have $\lim _{t \rightarrow \infty} x(t)=-\infty$, which contradicts the fact that $x$ is a positive solution of (1).

Second, we assume that (6) holds. Using the fact that $r \phi_{\gamma}\left(x^{\Delta}\right)$ is nonincreasing, we obtain, for $t \in\left[T_{1}, \infty\right)_{\mathbb{T}}$,

$$
\begin{aligned}
-x(t) & <\int_{t}^{\infty} \phi_{\gamma}^{-1}\left[r(u) \phi_{\gamma}\left(x^{\Delta}(u)\right)\right] r^{-1 / \gamma}(u) \Delta u \\
& \leq \phi_{\gamma}^{-1}\left[r(t) \phi_{\gamma}\left(x^{\Delta}(t)\right)\right] \int_{t}^{\infty} r^{-1 / \gamma}(u) \Delta u \\
& \leq \phi_{\gamma}^{-1}\left[r\left(T_{1}\right) \phi_{\gamma}\left(x^{\Delta}\left(T_{1}\right)\right)\right] \int_{t}^{\infty} r^{-1 / \gamma}(u) \Delta u \\
& =L_{1} \lambda(t)
\end{aligned}
$$

where $L_{1}:=\phi_{\gamma_{1}}^{-1}\left[r\left(T_{1}\right) \phi_{\gamma}\left(x^{\Delta}\left(T_{1}\right)\right)\right]<0$. By choosing sufficiently large $T_{2} \in\left[T_{1}, \infty\right)_{\mathbb{T}}$ such that $g_{0}(t) \geq T_{1}$ and $g(t, s) \geq T_{1}$, for $t \geq T_{2}$ and $s \in[a, b]_{\widehat{\mathbb{\pi}}}$, we get, for $t \geq T_{2}$ and $s \in[a, b]_{\widehat{\mathbb{N}}}$,

$$
\begin{gathered}
{\left[x\left(g_{0}(t)\right)\right]^{\gamma}>L \lambda^{\gamma}\left(g_{0}(t)\right),} \\
{[x(g(t, s))]^{\alpha(s)}>L \lambda^{\alpha(s)}(g(t, s)),}
\end{gathered}
$$

where $L:=\inf _{s \in[a, b]_{\hat{\mathbb{T}}}}\left\{-L_{1}^{\gamma},-L_{1}^{\alpha(s)}\right\}>0$. From (1) and (12) we find that

$$
\begin{aligned}
{\left[r(t) \phi_{\gamma}\left(x^{\Delta}(t)\right)\right]^{\Delta}<} & -L q_{0}(t) \lambda^{\gamma}\left(g_{0}(t)\right) \\
& -L \int_{a}^{b} q(t, s)\left[\lambda^{\alpha(s)}(g(t, s))\right] \Delta \zeta(s) \\
= & -L Q_{1}(t)
\end{aligned}
$$

Integrating this last inequality from $T_{2}$ to $t$, we see that

$$
\begin{aligned}
r(t) & \phi_{\gamma}\left(x^{\Delta}(t)\right) \\
& \leq r(t) \phi_{\gamma}\left(x^{\Delta}(t)\right)-r\left(T_{2}\right) \phi_{\gamma}\left(x^{\Delta}\left(T_{2}\right)\right) \\
& <-L \int_{T_{2}}^{t} Q_{1}(w) \Delta w
\end{aligned}
$$


which implies

$$
x^{\Delta}(t)<-r^{-1 / \gamma}(t)\left[L \int_{T_{2}}^{t} Q_{1}(u) \Delta u\right]^{1 / \gamma} .
$$

Again, integrating this last inequality from $T_{2}$ to $t$, we get

$$
x(t)-x\left(T_{2}\right)<-\int_{T_{2}}^{t} r^{-1 / \gamma}(v)\left[L \int_{T_{2}}^{v} Q_{1}(u) \Delta u\right]^{1 / \gamma} \Delta v .
$$

From (6), we have $\lim _{t \rightarrow \infty} x(t)=-\infty$, which contradicts the fact that $x$ is a positive solution of (1). This completes the proof.

Lemma 2. Assume that there exists sufficiently large $T \geq t_{0}$ such that

$$
\begin{array}{r}
x(t)>0, \quad x^{\Delta}(t)>0, \\
{\left[r(t) \phi_{\gamma}\left(x^{\Delta}(t)\right)\right]^{\Delta} \leq 0,} \\
t \in[T, \infty)_{\mathbb{T}} .
\end{array}
$$

Then

$$
\begin{gathered}
x\left(g_{0}(t)\right) \geq \varphi_{1}(t) x(t), \\
x(g(t, s)) \geq \varphi_{2}(t, s) x(t), \\
t \geq T_{1} \geq T,
\end{gathered}
$$

where

$$
\begin{gathered}
\varphi_{1}(t):= \begin{cases}1, & g_{0}(t) \geq t, \\
\frac{R\left(g_{0}(t), T\right)}{R(t, T)}, & g_{0}(t) \leq t,\end{cases} \\
\varphi_{2}(t, s):= \begin{cases}1, & g(t, s) \geq t, \\
\frac{R(g(t, s), T)}{R(t, T)}, & g(t, s) \leq t .\end{cases}
\end{gathered}
$$

Proof. Since $r \phi_{\gamma}\left(x^{\Delta}\right)$ is strictly decreasing on $[T, \infty)_{\mathbb{T}}$. If $\tau \geq$ $t$, then $x(\tau)>x(t)$ by the fact that $x$ is strictly increasing. Now we consider the case when $T \leq \tau \leq t$. We first have

$$
\begin{aligned}
x(t)-x(\tau) & =\int_{\tau}^{t} x^{\Delta}(s) \Delta s \\
& =\int_{\tau}^{t}\left[r(s) \phi_{\gamma}\left(x^{\Delta}(s)\right)\right]^{1 / \gamma} r^{-1 / \gamma}(s) \Delta s \\
& \leq\left[r(\tau) \phi_{\gamma}\left(x^{\Delta}(\tau)\right)\right]^{1 / \gamma} \int_{\tau}^{t} r^{-1 / \gamma}(s) \Delta s \\
& =\left[r(\tau) \phi_{\gamma}\left(x^{\Delta}(\tau)\right)\right]^{1 / \gamma} R(t, g(t, s)),
\end{aligned}
$$

which implies

$$
\frac{x(t)}{x(\tau)} \leq 1+\frac{\left[r(\tau) \phi_{\gamma}\left(x^{\Delta}(\tau)\right)\right]^{1 / \gamma}}{x(\tau)} R(t, g(t, s)) .
$$

On the other hand, we have

$$
\begin{aligned}
x(\tau) & >x(\tau)-x(T) \\
& =\int_{T}^{\tau}\left[r(s) \phi_{\gamma}\left(x^{\Delta}(s)\right)\right]^{1 / \gamma} r^{-1 / \gamma}(s) \Delta s \\
& \geq\left[r(\tau) \phi_{\gamma}\left(x^{\Delta}(\tau)\right)\right]^{1 / \gamma} \int_{T}^{\tau} r^{-1 / \gamma}(s) \Delta s \\
& =\left[r(\tau) \phi_{\gamma}\left(x^{\Delta}(\tau)\right)\right]^{1 / \gamma} R(\tau, T) .
\end{aligned}
$$

It implies that

$$
\frac{\left[r(\tau) \phi_{\gamma}\left(x^{\Delta}(\tau)\right)\right]^{1 / \gamma}}{x(\tau)} \leq \frac{1}{R(\tau, T)} .
$$

Therefore, (22) and (24) yield that

$$
\frac{x(t)}{x(\tau)} \leq 1+\frac{R(t, \tau)}{R(\tau, T)}=\frac{R(t, T)}{R(\tau, T)}
$$

and hence

$$
x(\tau) \geq \frac{R(\tau, T)}{R(t, T)} x(t), \quad t \geq T .
$$

Let $T_{1} \geq T$ so that $g_{0}(t)>T$ and $g(t, s)>T$ for $t \geq T_{1}$ and $s \in[a, b]_{\mathbb{\pi}}$. Thus, we have that, for $t \geq T_{1}$,

$$
x\left(g_{0}(t)\right) \geq \varphi_{1}(t) x(t), \quad x(g(t, s)) \geq \varphi_{2}(t, s) x(t) .
$$

This completes the proof.

We denote by $L_{\zeta}(a, b)_{\hat{\pi}}$ the set of Riemann-Stieltjes integrable functions on $[a, b)_{\hat{\pi}}$ with respect to $\zeta$. Let $b \in$ $[a, b)_{\hat{\pi}}$ such that $\alpha(c)=\gamma$. We further assume that

$$
\alpha, \alpha^{-1} \in L_{\zeta}(a, b)_{\widehat{\mathbb{N}}}
$$

such that

$$
\int_{a}^{c} \Delta \zeta(s)>0, \quad \int_{c}^{b} \Delta \zeta(s)>0 .
$$

We start with the following two lemmas cited from [25] which will play an important role in the proofs of our results.

Lemma 3. Let

$$
\begin{aligned}
& m:=\gamma \int_{\sigma(c)}^{b} \alpha^{-1}(s) \Delta \zeta(s)\left(\int_{\sigma(c)}^{b} \Delta \zeta(s)\right)^{-1}, \\
& n:=\gamma \int_{a}^{\sigma(c)} \alpha^{-1}(s) \Delta \zeta(s)\left(\int_{a}^{\sigma(c)} \Delta \zeta(s)\right)^{-1} .
\end{aligned}
$$

Then there exists $\eta \in L_{\zeta}(a, b)_{\widehat{\pi}}$ such that $\eta(s)>0$ on $[a, b)_{\widehat{\pi}}$,

$$
\int_{a}^{b} \alpha(s) \eta(s) \Delta \zeta(s)=\gamma, \quad \int_{a}^{b} \eta(s) \Delta \zeta(s)=1 .
$$


Lemma 4. Let $u \in C[a, b)_{\widehat{\pi}}$ and $\eta \in L_{\zeta}(a, b)_{\widehat{\mathbb{T}}}$ satisfying $u>0$, $\eta>0$ on $[a, b)_{\widehat{\pi}}$ and $\int_{a}^{b} \eta(s) \Delta \zeta(s)=1$. Then

$$
\int_{a}^{b} \eta(s) u(s) \Delta \zeta(s) \geq \exp \left(\int_{a}^{b} \eta(s) \ln [u(s)] \Delta \zeta(s)\right)
$$

where we use the convention that $\ln 0=-\infty$ and $e^{-\infty}=0$.

Theorem 5. Assume that one of conditions (5) and (6) holds. Furthermore, suppose that there exists a positive $\Delta$ differentiable function $\delta(t)$ such that, for all sufficiently large $T$,

$$
\limsup _{t \rightarrow \infty} \int_{T}^{t}\left[\delta(u) Q_{2}(u)-\frac{r(u)\left(\left(\delta^{\Delta}(u)\right)_{+}\right)^{\gamma+1}}{(\gamma+1)^{\gamma+1} \delta^{\gamma}(u)}\right] \Delta u=\infty,
$$

where

$$
\begin{aligned}
Q_{2}(u):= & q_{0}(u) \varphi_{1}^{\gamma}(u) \\
& +\exp \left(\int_{a}^{b} \eta(s) \ln \left[\frac{q(u, s) \varphi_{2}^{\alpha(s)}(u, s)}{\eta(s)}\right] \Delta \zeta(s)\right),
\end{aligned}
$$

with $\varphi_{1}$ and $\varphi_{2}$ being defined by (19) and (20), respectively. Then every solution of (1) is oscillatory.

Proof. Assume (1) has a nonoscillatory solution on $\left[t_{0}, \infty\right)_{\mathbb{T}}$. Then, without loss of generality, there is $T \in\left[t_{0}, \infty\right)_{\mathbb{T}}$, sufficiently large, so that $x(t)>0$ and $x(g(t, s))>0$ on $[T, \infty)_{\mathbb{T}} \times[a, b]_{\widehat{\mathbb{T}}}$. By Lemma 1 , we have, for $t \in[T, \infty)_{\mathbb{\mathbb { T }}}$,

$$
x^{\Delta}(t)>0, \quad\left[r(t) \phi_{\gamma}\left(x^{\Delta}(t)\right)\right]^{\Delta}<0, \quad t \geq T .
$$

Define

$$
w(t)=\delta(t) \frac{r(t) \phi_{\gamma}\left(x^{\Delta}(t)\right)}{\phi_{\gamma}(x(t))}
$$

By the product rule and the quotient rule, we have that

$$
\begin{aligned}
w^{\Delta}(t)= & {\left[\frac{\delta(t)}{\phi_{\gamma}(x(t))}\right]^{\Delta}\left[r(t) \phi_{\gamma}\left(x^{\Delta}(t)\right)\right]^{\sigma} } \\
& +\frac{\delta(t)}{\phi_{\gamma}(x(t))}\left[r(t) \phi_{\gamma}\left(x^{\Delta}(t)\right)\right]^{\Delta} \\
= & {\left[\frac{\delta^{\Delta}(t)}{\phi_{\gamma}\left(x^{\sigma}(t)\right)}-\frac{\delta(t)\left(x^{\gamma}(t)\right)^{\Delta}}{\phi_{\gamma}(x(t)) \phi_{\gamma}\left(x^{\sigma}(t)\right)}\right] } \\
& \times\left[r(t) \phi_{\gamma}\left(x^{\Delta}(t)\right)\right]^{\sigma}
\end{aligned}
$$

$$
\begin{aligned}
& +\frac{\delta(t)}{\phi_{\gamma}(x(t))}\left[r(t) \phi_{\gamma}\left(x^{\Delta}(t)\right)\right]^{\Delta} \\
= & \delta^{\Delta}(t)\left[\frac{r(t) \phi_{\gamma}\left(x^{\Delta}(t)\right)}{\phi_{\gamma}(x(t))}\right]^{\sigma} \\
& -\delta(t) \frac{\left(x^{\gamma}(t)\right)^{\Delta}}{x^{\gamma}(t)}\left[\frac{r(t) \phi_{\gamma}\left(x^{\Delta}(t)\right)}{\phi_{\gamma}(x(t))}\right]^{\sigma} \\
+ & \delta(t) \frac{\left[r(t) \phi_{\gamma}\left(x^{\Delta}(t)\right)\right]^{\Delta}}{\phi_{\gamma}(x(t))} .
\end{aligned}
$$

From (1) and the definition of $w(t)$, we have

$$
\begin{aligned}
w^{\Delta}(t)= & -\delta(t) \int_{a}^{b} q(t, s) \frac{[x(g(t, s))]^{\alpha(s)}}{x^{\gamma}(t)} \Delta \zeta(s) \\
& +\frac{\delta^{\Delta}(t)}{\delta^{\sigma}(t)} w^{\sigma}(t)-\frac{\delta(t)}{\delta^{\sigma}(t)} \frac{\left(x^{\gamma}(t)\right)^{\Delta}}{x^{\gamma}(t)} w^{\sigma}(t) .
\end{aligned}
$$

By the Pötzsche chain rule [3, Theorem 1.90], we obtain

$$
\begin{aligned}
\left(x^{\gamma}(t)\right)^{\Delta} & =\gamma \int_{0}^{1}\left[x(t)+h \mu(t) x^{\Delta}(t)\right]^{\gamma-1} d h x^{\Delta}(t) \\
& =\gamma \int_{0}^{1}\left[(1-h) x(t)+h x^{\sigma}(t)\right]^{\gamma-1} d h x^{\Delta}(t) \\
& \geq \begin{cases}\gamma(x(t))^{\gamma-1} x^{\Delta}(t), & \gamma \geq 1 \\
\gamma\left(x^{\sigma}(t)\right)^{\gamma-1} x^{\Delta}(t), & 0<\gamma \leq 1 .\end{cases}
\end{aligned}
$$

If $0<\gamma \leq 1$, we have that

$$
\begin{aligned}
w^{\Delta}(t) \leq & -\delta(t)\left[\frac{x\left(g_{0}(t)\right)}{x(t)}\right]^{\gamma} \\
& -\delta(t) \int_{a}^{b} q(t, s) \frac{[x(g(t, s))]^{\alpha(s)}}{x^{\gamma}(t)} \Delta \zeta(s) \\
& +\frac{\delta^{\Delta}(t)}{\delta^{\sigma}(t)} w^{\sigma}(t)-\frac{\gamma \delta(t)}{\delta^{\sigma}(t)} \frac{x^{\Delta}(t)}{x^{\sigma}(t)}\left(\frac{x^{\sigma}(t)}{x(t)}\right)^{\gamma} w^{\sigma}(t),
\end{aligned}
$$

whereas if $\gamma \geq 1$, we have that

$$
\begin{aligned}
w^{\Delta}(t) \leq & -\delta(t)\left[\frac{x\left(g_{0}(t)\right)}{x(t)}\right]^{\gamma} \\
& -\delta(t) \int_{a}^{b} q(t, s) \frac{[x(g(t, s))]^{\alpha(s)}}{x^{\gamma}(t)} \Delta \zeta(s) \\
& +\frac{\delta^{\Delta}(t)}{\delta^{\sigma}(t)} w^{\sigma}(t)-\frac{\gamma \delta(t)}{\delta^{\sigma}(t)} \frac{x^{\Delta}(t)}{x^{\sigma}(t)} \frac{x^{\sigma}(t)}{x(t)} w^{\sigma}(t) .
\end{aligned}
$$

Using the fact that $x(t)$ is strictly increasing and $r(t)\left(x^{\Delta}(t)\right)^{\gamma}$ is nonincreasing, we get that

$$
x^{\sigma}(t) \geq x(t), \quad x^{\Delta}(t) \geq\left(\frac{r^{\sigma}(t)}{r(t)}\right)^{1 / \gamma}\left(x^{\Delta}(t)\right)^{\sigma} .
$$


From (40), (41), and (42), we obtain

$$
\begin{aligned}
w^{\Delta}(t) \leq & -\delta(t)\left[\frac{x\left(g_{0}(t)\right)}{x(t)}\right]^{\gamma} \\
& -\delta(t) \int_{a}^{b} q(t, s) \frac{[x(g(t, s))]^{\alpha(s)}}{x^{\gamma}(t)} \Delta \zeta(s) \\
& +\frac{\left(\delta^{\Delta}(t)\right)_{+}}{\delta^{\sigma}(t)} w^{\sigma}(t)-\frac{\gamma \delta(t)\left(w^{\sigma}(t)\right)^{\lambda}}{\left(\delta^{\sigma}(t)\right)^{\lambda} r^{1 / \gamma}(t)}
\end{aligned}
$$

where $\lambda:=(\gamma+1) / \gamma$. By (18) and the definition of $\check{q}(t, s)$, we have that, for $t \geq T_{2}$ and $s \in[a, b]_{\widehat{\mathbb{T}}}$,

$$
\begin{gathered}
w^{\Delta}(t) \leq-\delta(t) q_{1}(t)-\delta(t) \int_{a}^{b} q_{2}(t, s) x^{\alpha(s)-\gamma}(t) \Delta \zeta(s) \\
+\frac{\left(\delta^{\Delta}(t)\right)_{+}}{\delta^{\sigma}(t)} w^{\sigma}(t)-\frac{\gamma \delta(t)\left(w^{\sigma}(t)\right)^{\lambda}}{\left(\delta^{\sigma}(t)\right)^{\lambda} r^{1 / \gamma}(t)}
\end{gathered}
$$

where $q_{1}(t):=q_{0}(t) \varphi_{1}^{\gamma}(t)$ and $q_{2}(t, s):=q(t, s) \varphi^{\alpha(s)}(t, s)$. We let $\eta \in L_{\zeta}(a, b)_{\hat{\pi}}$ be defined as in Lemma 3. Then $\eta$ satisfies (31). This follows the fact that

$$
\int_{a}^{b} \eta(s)[\alpha(s)-\gamma] \Delta \zeta=0
$$

From Lemma 4 we get

$$
\begin{aligned}
& \int_{a}^{b} q_{2}(t, s)[x(t)]^{\alpha(s)-\gamma} \Delta \zeta(s) \\
& =\int_{a}^{b} \eta(s) \frac{q_{2}(t, s)}{\eta(s)}[x(t)]^{\alpha(s)-\gamma} \Delta \zeta(s) \\
& \geq \exp \left(\int_{a}^{b} \eta(s) \ln \left(\frac{q_{2}(t, s)}{\eta(s)}[x(t)]^{\alpha(s)-\gamma}\right) \Delta \zeta(s)\right) \\
& =\exp \left(\int_{a}^{b} \eta(s) \ln \left[\frac{q_{2}(t, s)}{\eta(s)}\right] \Delta \zeta(s)\right. \\
& \left.\quad+\ln (x(t)) \int_{a}^{b} \eta(s)[\alpha(s)-\gamma] \Delta \zeta(s)\right) \\
& =\exp \left(\int_{a}^{b} \eta(s) \ln \left[\frac{q_{2}(t, s)}{\eta(s)}\right] \Delta \zeta(s)\right) .
\end{aligned}
$$

This together with (44) shows that, for $t \geq T_{2}$,

$$
w^{\Delta}(t) \leq-\delta(t) Q_{2}(t)+\frac{\left(\delta^{\Delta}(t)\right)_{+}}{\delta^{\sigma}(t)} w^{\sigma}(t)-\frac{\gamma \delta(t)\left(w^{\sigma}(t)\right)^{\lambda}}{\left(\delta^{\sigma}(t)\right)^{\lambda} r^{1 / \gamma}(t)} .
$$

Define $A \geq 0$ and $B \geq 0$ by

$$
A^{\lambda}:=\frac{\gamma \delta(t)\left(w^{\sigma}(t)\right)^{\lambda}}{\left(\delta^{\sigma}(t)\right)^{\lambda} r^{1 / \gamma}(t)}, \quad B^{\lambda-1}:=\frac{\left(r^{1 / \lambda}(t)\right)^{1 / \lambda}\left(\delta^{\Delta}(t)\right)_{+}}{\lambda \gamma^{1 / \lambda}(\delta(t))^{1 / \lambda}} .
$$

Then, using the inequality [27]

$$
\lambda A B^{\lambda-1}-A^{\lambda} \leq(\lambda-1) B^{\lambda},
$$

we get that

$$
\frac{\left(\delta^{\Delta}(t)\right)_{+}}{\delta^{\sigma}(t)} w^{\sigma}(t)-\frac{\gamma \delta(t)\left(w^{\sigma}(t)\right)^{\lambda}}{\left(\delta^{\sigma}(t)\right)^{\lambda} r^{1 / \gamma}(t)} \leq \frac{r(t)\left(\left(\delta^{\Delta}(t)\right)_{+}\right)^{\gamma+1}}{(\gamma+1)^{\gamma+1} \delta^{\gamma}(t)} .
$$

From this last inequality and (47) we get, for $t \geq T_{2}$,

$$
w^{\Delta}(t) \leq-\delta(t) Q_{2}(t)+\frac{r(t)\left(\left(\delta^{\Delta}(t)\right)_{+}\right)^{\gamma+1}}{(\gamma+1)^{\gamma+1} \delta^{\gamma}(t)} .
$$

Integrating both sides from $T_{2}$ to $t$, we get

$$
\begin{aligned}
& \int_{T_{2}}^{t}\left[\delta(u) Q_{2}(u)-\frac{r(u)\left(\left(\delta^{\Delta}(u)\right)_{+}\right)^{\gamma+1}}{(\gamma+1)^{\gamma+1} \delta^{\gamma}(u)}\right] \Delta u \\
& \leq w\left(T_{2}\right)-w(t) \leq w\left(T_{2}\right),
\end{aligned}
$$

which leads to a contradiction to (33).

In the following examples, for $\widehat{\mathbb{T}}=\mathbb{R}, n \in \mathbb{N}$, and $s \in$ $[0, n+1)$, we assume that

$$
\zeta(s)=\sum_{j=1}^{n} \chi(s-j) \quad \text { with } \chi(s)= \begin{cases}1, & s \geq 0 \\ 0, & s<0\end{cases}
$$

$\alpha \in C[0, n+1)$ such that $\alpha(j)=\alpha_{j}, j=1, \ldots, n$,

$$
\begin{gathered}
\alpha_{j}>\gamma, \quad j=1,2, \ldots, l, \\
\alpha_{j}<\gamma, \quad j=l+1, l+2, \ldots, n
\end{gathered}
$$

$q(t, j)=q_{j}(t)$ and $g(t, j)=g_{j}(t)$ for $j=1, \ldots, n$.

Example 6. Consider the nonlinear dynamic equation

$$
\begin{aligned}
& {\left[t^{\gamma-1} \phi_{\gamma}\left(x^{\Delta}(t)\right)\right]^{\Delta}+\frac{1}{t^{1 /(\gamma+1)}} x^{\gamma}\left(g_{0}(t)\right)} \\
& \quad+\sum_{j=1}^{n} q_{j}(t) \phi_{\alpha_{j}}\left(x\left(g_{j}(t)\right)\right)=0, \quad t \in\left[t_{0}, \infty\right)_{\mathbb{T}},
\end{aligned}
$$

where $g_{j}, j=0,1,2, \ldots, n$, are rd-continuous functions with $g_{0}(t) \geq t$ on $\left[t_{0}, \infty\right)_{\mathbb{T}}, \gamma$ and $\alpha_{j}, j=1,2, \ldots, n$, are positive constants, and $q_{j}, j=1,2, \ldots, n$, are nonnegative rdcontinuous functions on $\mathbb{T}$. Here,

$$
r(t)=t^{\gamma-1}, \quad q_{0}(t)=\frac{1}{t^{1 /(\gamma+1)}} .
$$

Choose an $n$-tuple $\left(\eta_{1}, \eta_{2}, \ldots, \eta_{n}\right)$ with $0<\eta_{j}<1$ satisfying (31). By Example 5.60 in [4], condition (5) holds since

$$
\int_{t_{0}}^{\infty} r^{-1 / \gamma}(t) \Delta t=\int_{t_{0}}^{\infty} \frac{\Delta t}{t^{1-1 / \gamma}}=\infty
$$


Also, by choosing $\delta(t) \equiv 1$, we have

$$
\begin{gathered}
\limsup _{t \rightarrow \infty} \int_{T}^{t}\left[\delta(u) Q_{2}(u)-\frac{r(u)\left(\left(\delta^{\Delta}(u)\right)_{+}\right)^{\gamma+1}}{(\gamma+1)^{\gamma+1} \delta^{\gamma}(u)}\right] \Delta u \\
\quad \geq \limsup _{t \rightarrow \infty} \int_{T}^{t} \frac{1}{u^{1 /(\gamma+1)}} \Delta u=\infty .
\end{gathered}
$$

Then, by Theorem 5, every solution of (55) is oscillatory.

Example 7. Consider the nonlinear dynamic equation

$$
\begin{aligned}
& {\left[(t \sigma(t))^{\gamma} \phi_{\gamma}\left(x^{\Delta}(t)\right)\right]^{\Delta}} \\
& \quad+\sum_{j=0}^{n} q_{j}(t) \phi_{\alpha_{j}}\left(x\left(g_{j}(t)\right)\right)=0, \quad t \in\left[t_{0}, \infty\right)_{\mathbb{T}},
\end{aligned}
$$

where $0<\gamma=\alpha_{0} \leq 1$ is a positive real number, $q_{0}(t):=t^{\gamma}$, $\alpha_{j}, j=1,2, \ldots, n$, are positive constants, $q_{j}, j=1,2, \ldots, n$, are nonnegative rd-continuous functions on $\mathbb{T}$, and $g_{j}, j=$ $0,1,2, \ldots, n$, are rd-continuous functions with $g_{0}(t) \leq t$ on $\left[t_{0}, \infty\right)_{\mathbb{T}}$. Assume

$$
\int_{t_{0}}^{\infty} \frac{\Delta t}{t^{1-1 / \alpha_{0}} \sigma(t)}=\infty, \quad 0<\alpha_{0} \leq 1
$$

It is clear that $r(t)$ satisfies

$$
\begin{aligned}
& \int_{t_{0}}^{\infty} r^{-1 / \gamma}(t) \Delta t<\infty \leq \int_{t_{0}}^{\infty} \frac{1}{t \sigma(t)} \Delta t=\int_{t_{0}}^{\infty}\left(\frac{-1}{t}\right)^{\Delta} \Delta t<\infty, \\
& t \in\left[t_{0}, \infty\right)_{\mathbb{T}}, t_{0}>0 .
\end{aligned}
$$

This holds for many time scales, for example, when $\mathbb{T}=q^{\mathbb{N}_{0}}=$ $\left\{t: t=q^{k}, k \in \mathbb{N}_{0}, q>1\right\}$. To see that (6) holds note that

$$
\begin{aligned}
& \int_{t_{0}}^{\infty} r^{-1 / \gamma}(v)\left[\int_{t_{0}}^{v} Q_{1}(u) \Delta u\right]^{1 / \gamma} \Delta v \\
& \quad=\int_{t_{0}}^{\infty} r^{-1 / \alpha_{0}}(v)\left[\int_{t_{0}}^{v} \sum_{j=0}^{n} q_{j}(u) \lambda^{\alpha_{j}}\left(g_{j}(u)\right) \Delta u\right]^{1 / \alpha_{0}} \Delta v \\
& \quad \geq \int_{t_{0}}^{\infty} \frac{1}{v \sigma(v)}\left[\int_{t_{0}}^{v} u^{\alpha_{0}} \lambda^{\alpha_{0}}\left(g_{0}(u)\right) \Delta u\right]^{1 / \alpha_{0}} \Delta v \\
& \quad \geq \int_{t_{0}}^{\infty} \frac{\left(v-t_{0}\right)^{1 / \alpha_{0}}}{v \sigma(v)} \Delta v .
\end{aligned}
$$

Since

$$
\begin{aligned}
\lambda\left(g_{0}(u)\right) & =\int_{g_{0}(u)}^{\infty} r^{-1 / \gamma}(w) \Delta w=\int_{g_{0}(u)}^{\infty} \frac{1}{w \sigma(w)} \Delta w \\
& =\int_{g_{0}(u)}^{\infty}\left(\frac{-1}{w}\right)^{\Delta} \Delta w=\frac{1}{g_{0}(u)} \geq \frac{1}{u},
\end{aligned}
$$

we can find $0<k<1$ such that $v-t_{0}>k v$ for $v \geq t_{k}>t_{0}$. Therefore, we get

$$
\begin{gathered}
\int_{t_{0}}^{\infty} r^{-1 / \gamma}(v)\left[\int_{t_{0}}^{v} Q_{1}(u) \Delta u\right]^{1 / \gamma} \Delta v \\
>k^{1 / \alpha_{0}} \int_{t_{K}}^{\infty} \frac{\Delta v}{v^{1-1 / \alpha_{0}} \sigma(v)} \stackrel{(60)}{=} \infty .
\end{gathered}
$$

To apply Theorem 5, it remains to prove that condition (33) holds. By putting $\delta(t) \equiv 1$, we get

$$
\begin{gathered}
\limsup _{t \rightarrow \infty} \int_{T}^{t}\left[\delta(u) Q_{2}(u)-\frac{r(u)\left(\left(\delta^{\Delta}(u)\right)_{+}\right)^{\gamma+1}}{(\gamma+1)^{\gamma+1} \delta^{\gamma}(u)}\right] \Delta u \\
\quad \geq \limsup _{t \rightarrow \infty} \int_{T}^{t} u^{\gamma} \Delta u=\infty .
\end{gathered}
$$

We conclude that if $\left[t_{0}, \infty\right)_{\mathbb{T}}, t_{0}>0$, is a time scale, where $\int_{t_{0}}^{\infty}\left(\Delta t / t^{1-1 / \gamma} \sigma(t)\right)=\infty$, then every solution of (59) is oscillatory by Theorem 5 .

We are now ready to state and prove Philos-type oscillation criteria for (1). Its proof can be similarly done as [28] and hence is omitted.

Theorem 8. Assume that one of conditions (5) and (6) holds. Furthermore, suppose that there exist functions $H, h \in$ $C_{r d}(\mathbb{D}, \mathbb{R})$, where $\mathbb{D} \equiv\left\{(t, u): t \geq u \geq t_{0}\right\}$ such that

$$
H(t, t)=0, \quad t \geq t_{0}, \quad H(t, u)>0, \quad t>u \geq t_{0},
$$

and $H$ has a nompositive continuous $\Delta$-partial derivative $H^{\Delta_{u}}(t, u)$ with respect to the second variable and satisfies

$$
H^{\Delta_{u}}(t, u)+H(t, u) \frac{\delta^{\Delta}(u)}{\delta^{\sigma}(u)}=-\frac{h(t, u)}{\delta^{\sigma}(u)}(H(t, u))^{\gamma /(\gamma+1)},
$$

and, for all sufficiently large $T$,

$$
\begin{aligned}
\limsup _{t \rightarrow \infty} \frac{1}{H(t, T)} \int_{T}^{t} & {\left[\delta(u) Q_{2}(u) H(t, u)\right.} \\
& \left.-\frac{\left(h_{-}(t, u)\right)^{\gamma+1} r(u)}{(\gamma+1)^{\gamma+1} \delta^{\gamma}(u)}\right] \Delta u=\infty,
\end{aligned}
$$

where $\delta(t)$ is a positive $\Delta$-differentiable function. Then every solution of (1) is oscillatory on $\left[t_{0}, \infty\right)_{\mathbb{T}}$.

Example 9. Consider the following dynamic equation:

$$
\begin{aligned}
& {\left[\phi_{\gamma}\left(x^{\Delta}(t)\right)\right]^{\Delta}+q_{0}(t) \phi_{\gamma}\left(g_{0}(t)\right)} \\
& \quad+\sum_{j=1}^{n} q_{j}(t) \phi_{\alpha_{j}}\left(x\left(g_{j}(t)\right)\right)=0, \quad t \in\left[t_{0}, \infty\right)_{\mathbb{T}},
\end{aligned}
$$


where $r(t)=1, g_{j}, q_{j}, j=0,1,2, \ldots, n$, are rd-continuous functions with $g_{0}(t) \geq t$ and $q_{j}(t) \geq 0$ on $t \in\left[t_{0}, \infty\right)_{T}$, and $\gamma$ and $\alpha_{j}, j=1,2, \ldots, n$, are positive constants. It is easy to see that (5) holds. Choose an $n$-tuple $\left(\eta_{1}, \eta_{2}, \ldots, \eta_{n}\right)$ with $0<\eta_{j}<$ 1 satisfying (31). By the definition of $\varphi_{1}$, we know $\varphi_{1}(t) \equiv 1$. On the other hand, let $H(t, u)=(t-u)^{2}$ and $\delta(t) \equiv 1$. From (67), we obtain

$$
H^{\Delta_{u}}(t, u)=\sigma(u)+u-2 t=-h(t, u)(H(t, u))^{\gamma /(\gamma+1)} .
$$

We have that $h(t, u) \geq 0$ for $u \in\left[t_{0}, t\right)_{\mathbb{T}}$ and hence $h_{-}(t, u) \equiv 0$ for $u \in\left[t_{0}, t\right)_{\mathbb{T}}$. Therefore,

$$
\begin{array}{r}
\limsup _{t \rightarrow \infty} \frac{1}{H(t, T)} \int_{T}^{t}\left[\delta(u) Q_{2}(u) H(t, u)\right. \\
\left.-\frac{\left(h_{-}(t, u)\right)^{\gamma+1} r(u)}{(\gamma+1)^{\gamma+1} \delta^{\gamma}(u)}\right] \Delta u \\
\geq \limsup _{t \rightarrow \infty} \frac{1}{(t-T)^{2}} \int_{T}^{t}\left[q_{0}(u)(t-u)^{2}\right] \Delta u .
\end{array}
$$

By Theorem 8, we can say that every solution of (69) is oscillatory if

$$
\limsup _{t \rightarrow \infty} \frac{1}{(t-T)^{2}} \int_{T}^{t}\left[q_{0}(u)(t-u)^{2}\right] \Delta u=+\infty .
$$

Theorem 10. Assume that one of conditions (5) and (6) holds and

$$
\limsup _{t \rightarrow \infty} R^{\gamma}(t, T) \int_{t}^{\infty} Q_{2}(u) \Delta u>1 .
$$

Then every solution of (18) is oscillatory.

Proof. Assume (1) has a nonoscillatory solution on $\left[t_{0}, \infty\right)_{\mathbb{T}}$. Then, without loss of generality, there is a $T \in\left[t_{0}, \infty\right)_{\mathbb{T}}$, sufficiently large, so that $x(t)>0$ and $x(g(t, s))>0$ on $[T, \infty)_{\mathbb{T}} \times[a, b]_{\widehat{\pi}}$. Then, by Lemma 1 , we have, for $t \in$ $[T, \infty)_{\mathbb{T}}$,

$$
x^{\Delta}(t)>0, \quad\left[r(t) \phi_{\gamma}\left(x^{\Delta}(t)\right)\right]^{\Delta}<0, \quad t \geq T .
$$

Integrating both sides of the dynamic equation (18) from $t$ to $\infty$, we obtain

$$
\begin{aligned}
& r(t) \phi_{\gamma}\left(x^{\Delta}(t)\right) \\
& \geq \int_{t}^{\infty} q_{0}(u) \phi_{\gamma}(x(h(u))) \Delta u \\
& +\int_{t}^{\infty} \int_{a}^{b} q(u, s) \phi_{\alpha(s)}(x(g(u, s))) \Delta \zeta(s) \Delta u \\
& \geq \int_{t}^{\infty} x^{\gamma}(u)\left\{q_{0}(u)\left[\frac{x(h(u))}{x(u)}\right]^{\gamma}\right. \\
& \left.\quad+\int_{a}^{b} q(u, s) \frac{[x(g(u, s))]^{\alpha(s)}}{x^{\gamma}(u)} \Delta \zeta(s)\right\} \Delta u .
\end{aligned}
$$

As shown in the proof of Theorem 5, we have

$$
\begin{gathered}
q_{0}(u)\left[\frac{x(h(u))}{x(u)}\right]^{\gamma}+\int_{a}^{b} q(u, s) \frac{[x(g(u, s))]^{\alpha(s)}}{x^{\gamma}(u)} \Delta \zeta(s) \\
\quad \geq Q_{2}(u) .
\end{gathered}
$$

Then, from (75) and (76), we get

$$
\begin{aligned}
r(t) \phi_{\gamma}\left(x^{\Delta}(t)\right) & \geq \int_{t}^{\infty} x^{\gamma}(u) Q(u) \Delta u \\
& \geq x^{\gamma}(t) \int_{t}^{\infty} Q_{2}(u) \Delta u .
\end{aligned}
$$

Since $x^{\Delta}(t)>0$ and $r(t)>0$, we have

$$
\frac{1}{r(t)} \int_{t}^{\infty} Q_{2}(u) \Delta u \leq\left[\frac{x^{\Delta}(t)}{x(t)}\right]^{\gamma} \text {. }
$$

Also, by using the fact that $r \phi_{\gamma}\left(x^{\Delta}\right)$ is nonincreasing, we have

$$
\begin{aligned}
x(t) & \geq x(t)-x(T)=\int_{T}^{t} x^{\Delta}(s) \Delta s \\
& =\int_{T}^{t}\left[r(s) \phi_{\gamma}\left(x^{\Delta}(s)\right)\right]^{1 / \gamma} r^{-1 / \gamma}(s) \Delta s \\
& \geq\left[r(t) \phi_{\gamma}\left(x^{\Delta}(t)\right)\right]^{1 / \gamma} \int_{T}^{t} r^{-1 / \gamma}(s) \Delta s \\
& =\left[r(t) \phi_{\gamma}\left(x^{\Delta}(t)\right)\right]^{1 / \gamma} R(t, T),
\end{aligned}
$$

or

$$
\left[\frac{x^{\Delta}(t)}{x(t)}\right]^{\gamma} \leq \frac{1}{r(t) R^{\gamma}(t, T)} .
$$

In view of (78) and (80), we get

$$
R^{\gamma}(t, T) \int_{t}^{\infty} Q_{2}(u) \Delta u \leq 1,
$$

which gives us the contradiction

$$
\limsup _{t \rightarrow \infty} R^{\gamma}(t, T) \int_{t}^{\infty} Q_{2}(u) \Delta u \leq 1 .
$$

This completes the proof.

Example 11. For $t \in\left[t_{0}, \infty\right)_{\mathbb{T}}$, we consider the following dynamic equation:

$$
\begin{array}{r}
{\left[\phi_{\gamma}\left(x^{\Delta}(t)\right)\right]^{\Delta}+\frac{1}{t \sigma(t)} \phi_{\gamma}\left(x\left(g_{0}(t)\right)\right)} \\
+\sum_{j=1}^{n} q_{j}(t) \phi_{\alpha_{j}}\left(x\left(g_{j}(t)\right)\right)=0,
\end{array}
$$

where $r(t)=1, q_{0}(t)=1 / t \sigma(t), g_{j}, j=0,1,2, \ldots, n$, are rdcontinuous functions with $g_{0}(t) \geq t$ on $t \in\left[t_{0}, \infty\right)_{\mathbb{T}}, q_{j}$, 
$j=1,2, \ldots, n$, are nonnegative rd-continuous functions on $\mathbb{T}, \gamma>1$, and $\alpha_{j}, j=1,2, \ldots, n$, are positive constants. It is obvious that $(5)$ holds. Choose an $n$-tuple $\left(\eta_{1}, \eta_{2}, \ldots, \eta_{n}\right)$ with $0<\eta_{j}<1$ satisfying (31). On the other hand, noting that $\varphi_{1}(t)=1$ and $R(t, T)=\int_{T}^{t} r^{-1 / \gamma}(s) \Delta s=t-T$, we can easily verify that

$$
\begin{aligned}
& \limsup _{t \rightarrow \infty} R^{\gamma}(t, T) \int_{t}^{\infty} Q_{2}(u) \Delta u \\
& \quad \geq \limsup _{t \rightarrow \infty}(t-T)^{\gamma} \int_{t}^{\infty} \frac{1}{u \sigma(u)} \Delta u=+\infty>1 .
\end{aligned}
$$

By Theorem 10, every solution of (83) is oscillatory.

The last theorem is under the assumption that $\int_{t_{0}}^{\infty} Q_{2}(u) \Delta u<\infty$. Its proof can be similarly done as in [28] and hence is omitted.

Theorem 12. Assume that one of conditions (5) and (6) holds and $r(t)$ is a (delta) differentiable function with $r^{\Delta}(t) \geq 0$. Furthermore, assume that $l=\liminf _{t \rightarrow \infty}(t / \sigma(t))>0$ and

$$
\liminf _{t \rightarrow \infty} \frac{t^{\gamma}}{r(t)} \int_{\sigma(t)}^{\infty} Q_{2}(u) \Delta u>\frac{\gamma^{\gamma}}{l \gamma^{2}(\gamma+1)^{\gamma+1}}
$$

Then every solution of (1) is oscillatory.

\section{Conflict of Interests}

The authors declare that there is no conflict of interests regarding the publication of this paper.

\section{Acknowledgments}

The authors thank the associate editor and the reviewers for their valuable comments on this paper. This work was supported by the Natural Science Foundation of Shandong Province under Grant nos. JQ201119 and ZR2010AL002 and the National Natural Science Foundation of China under Grant no. 61174217.

\section{References}

[1] V. Kac and P. Cheung, Quantum Calculus, Universitext, 2002.

[2] S. Hilger, "Analysis on measure chains-a unified approach to continuous and discrete calculus," Results in Mathematics, vol. 18, no. 1-2, pp. 18-56, 1990.

[3] M. Bohner and A. Peterson, Dynamic Equations on Time Scales: An Introduction with Applications, Birkhäauser, Boston, Mass, USA, 2001.

[4] M. Bohner and A. Peterson, Advances in Dynamic Equations on Time Scales, Birkhäauser, Boston, Mass, USA, 2003.

[5] R. P. Agarwal, D. R. Anderson, and A. Zafer, "Interval oscillation criteria for second-order forced delay dynamic equations with mixed nonlinearities," Computers \& Mathematics with Applications, vol. 59, no. 2, pp. 977-993, 2010.

[6] S. R. Grace, R. P. Agarwal, M. Bohner, and D. O’Regan, "Oscillation of second-order strongly superlinear and strongly sublinear dynamic equations," Communications in Nonlinear Science and Numerical Simulation, vol. 14, no. 8, pp. 3463-3471, 2009.

[7] R. P. Agarwal, S. R. Grace, and D. O'Regan, Oscillation Theory for Second Order Dynamic Equations, vol. 5, Taylor \& Francis, London, UK, 2003.

[8] R. Agarwal, M. Bohner, D. O’Regan, and A. Peterson, "Dynamic equations on time scales: a survey," Journal of Computational and Applied Mathematics, vol. 141, no. 1-2, pp. 1-26, 2002.

[9] O. Došlý and S. Hilger, "A necessary and sufficient condition for oscillation of the Sturm-Liouville dynamic equation on time scales," Journal of Computational and Applied Mathematics, vol. 141, no. 1-2, pp. 147-158, 2002.

[10] L. Erbe, A. Peterson, and S. H. Saker, "Oscillation criteria for second-order nonlinear dynamic equations on time scales," Journal of the London Mathematical Society, vol. 67, no. 3, pp. 701-714, 2003.

[11] T. S. Hassan, "Oscillation criteria for half-linear dynamic equations on time scales," Journal of Mathematical Analysis and Applications, vol. 345, no. 1, pp. 176-185, 2008.

[12] T. S. Hassan, "Kamenev-type oscillation criteria for second order nonlinear dynamic equations on time scales," Applied Mathematics and Computation, vol. 217, no. 12, pp. 5285-5297, 2011.

[13] T. S. Hassan, L. Erbe, and A. Peterson, "Oscillation of second order superlinear dynamic equations with damping on time scales," Computers \& Mathematics with Applications, vol. 59, no. 1, pp. 550-558, 2010.

[14] T. S. Hassan, L. Erbe, and A. Peterson, "Oscillation criteria for second order sublinear dynamic equations with damping term," Journal of Difference Equations and Applications, vol. 17, no. 4, pp. 505-523, 2011.

[15] Q. Lin, B. Jia, and Q. Wang, "Forced oscillation of secondorder half-linear dynamic equations on time scales," Abstract and Applied Analysis, vol. 2010, Article ID 294194, 10 pages, 2010.

[16] A. Özbekler and A. Zafer, "Oscillation of solutions of second order mixed nonlinear differential equations under impulsive perturbations," Computers \& Mathematics with Applications, vol. 61, no. 4, pp. 933-940, 2011.

[17] Y. Şahiner, "Oscillation of second-order delay differential equations on time scales," Nonlinear Analysis: Theory, Methods and Applications, vol. 63, no. 5-7, pp. e1073-e1080, 2005.

[18] S. H. Saker, "Oscillation criteria of second-order half-linear dynamic equations on time scales," Journal of Computational and Applied Mathematics, vol. 177, no. 2, pp. 375-387, 2005.

[19] R. P. Agarwal, D. O’Regan, and S. H. Saker, "Philos-type oscillation criteria for second order half-linear dynamic equations on time scales," The Rocky Mountain Journal of Mathematics, vol. 37, no. 4, pp. 1085-1398, 2007.

[20] S. H. Saker, D. O’Regan, and R. P. Agarwal, "Oscillation theorems for second-order nonlinear neutral delay dynamic equations on time scales," Acta Mathematica Sinica, vol. 24, no. 9, pp. 1409-1432, 2008.

[21] S. H. Saker and D. O’Regan, "New oscillation criteria for second-order neutral dynamic equations on time scales via Riccati substitution," Hiroshima Mathematical Journal, vol. 42, no. 1, pp. 77-98, 2012.

[22] D. R. Anderson and S. H. Saker, "Interval oscillation criteria for forced Emden-Fowler functional dynamic equations with oscillatory potential,' Science China Mathematics, vol. 56, no. 3, pp. 561-576, 2013. 
[23] H. Liu and P. Liu, "Oscillation criteria for some new generalized Emden-Fowler dynamic equations on time scales," Abstract and Applied Analysis, vol. 2013, Article ID 962590, 16 pages, 2013.

[24] Y. Sun and Q. Kong, "Interval criteria for forced oscillation with nonlinearities given by Riemann-Stieltjes integrals," Computers \& Mathematics with Applications, vol. 62, no. 1, pp. 243-252, 2011.

[25] Y. Sun, "Interval oscillation criteria for second-order dynamic equations with nonlinearities given by Riemann-Stieltjes integrals," Abstract and Applied Analysis, vol. 2011, Article ID 719628, 14 pages, 2011.

[26] L. Erbe, T. Hassan, and A. Peterson, "Oscillation of second order functional dynamic equations," International Journal of Difference Equations, vol. 5, no. 2, pp. 175-193, 2010.

[27] G. H. Hardy, J. E. Littlewood, and G. Pólya, Inequalities, Cambridge University Press, Cambridge, UK, 2nd edition, 1988.

[28] S. H. Saker, Oscillation Theory of Dynamic Equations on Time Scales: Second and Third Orders, LAP Lambert Academic Publishing, 2010. 


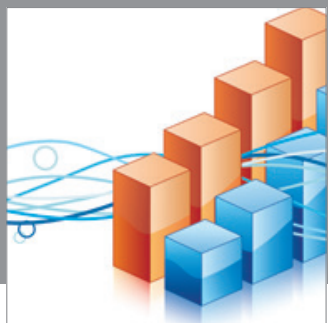

Advances in

Operations Research

mansans

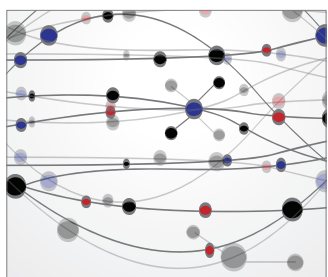

The Scientific World Journal
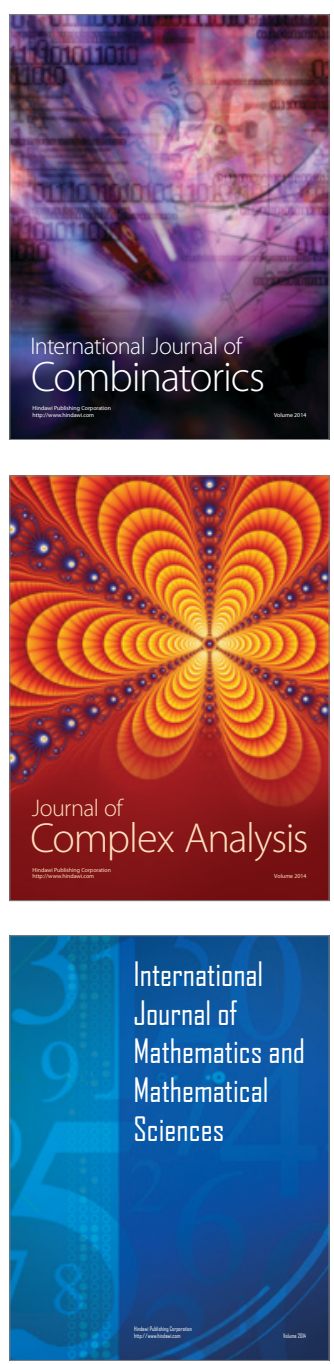
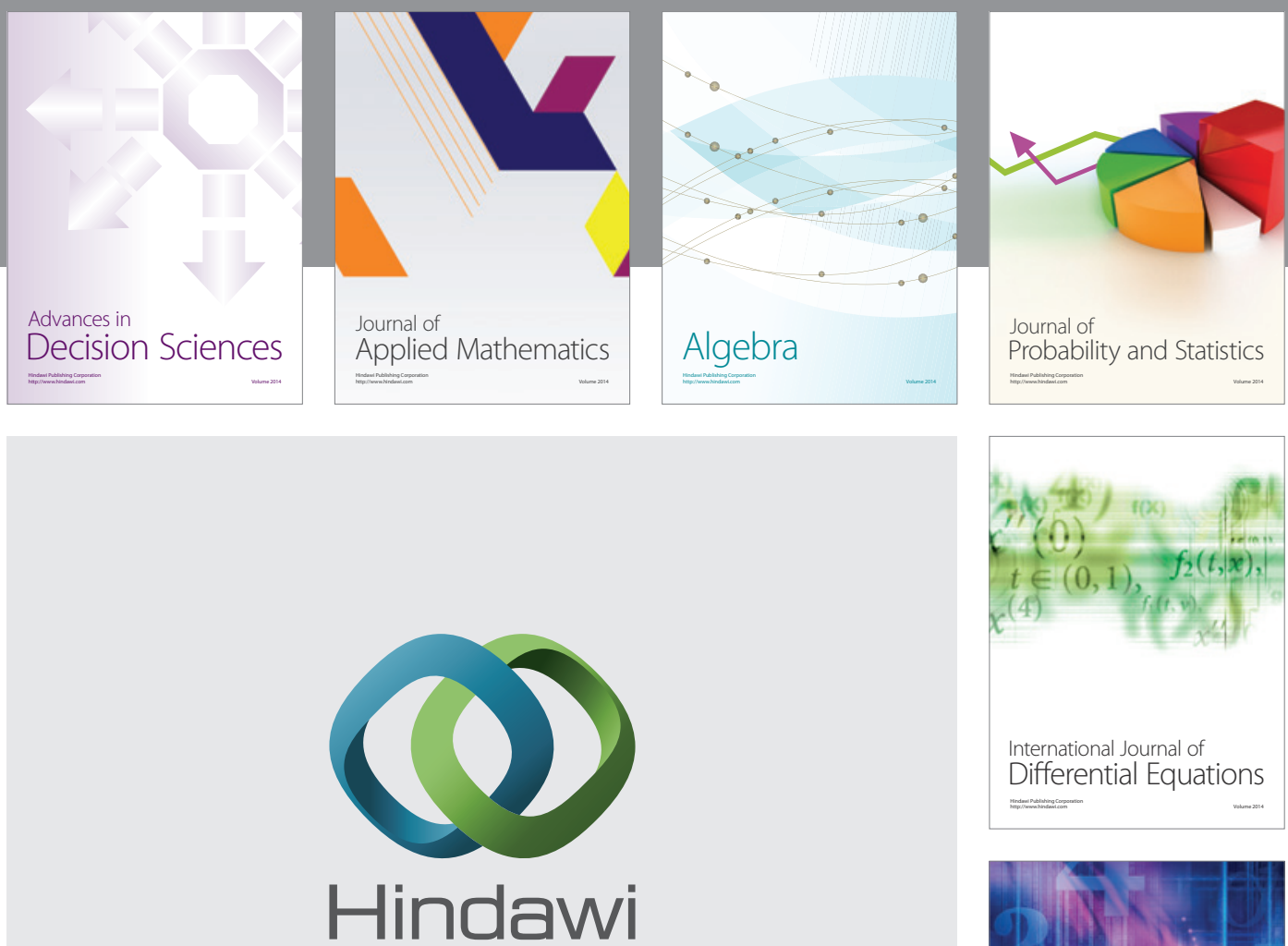

Submit your manuscripts at http://www.hindawi.com
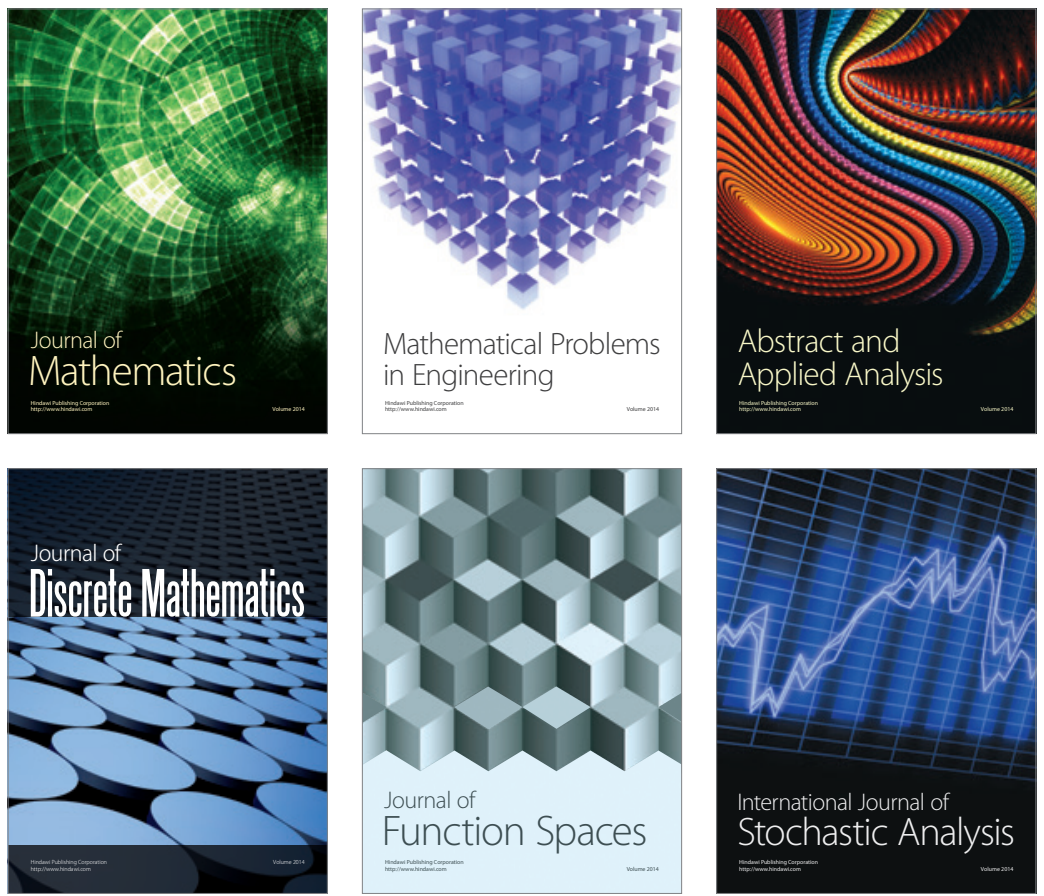

Journal of

Function Spaces

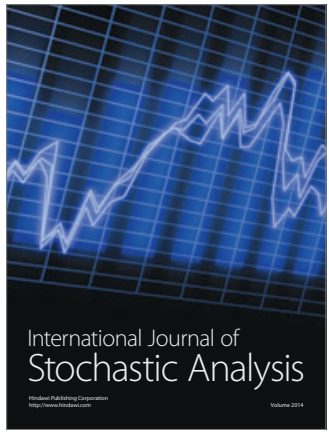

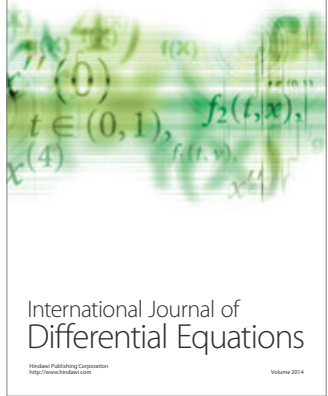
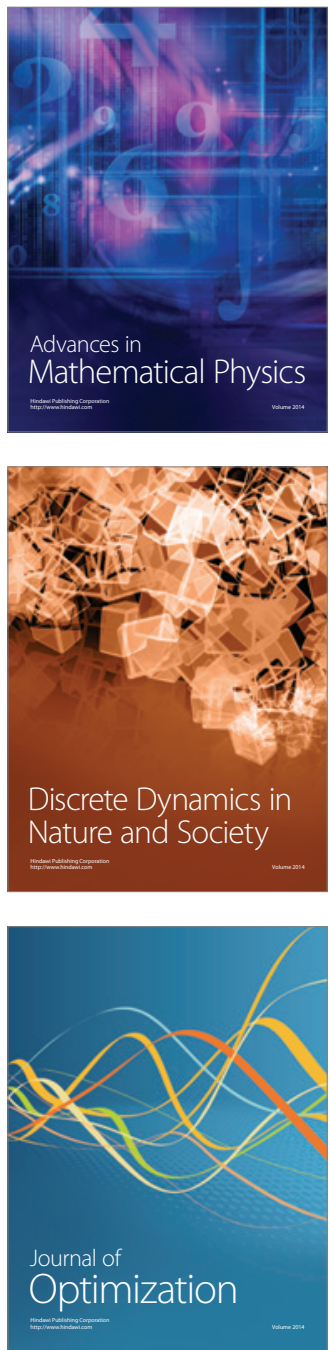